There seems to be a parallel between our observa. tions on the effect of sugars upon the extent of climb. ing of these algal filaments and those of Maige and Nicolas ${ }^{4}$ upon the effect of sugar solutions on the respiratory activity of various plant tissues. The latter showed that, during the first period of immersion in a sugar solution, a plant tissue showed a period of decreasing respiratory activity followed by one of increasing activity during the period of sugar penetration.

It is hoped to carry out further experiments to determine the effect of electrolytic and non-electrolytic solutions upon the rate of respiration of this alga, and also to develop a more accurate quantitative method of measuring the extent of the climbing phenomenon.

Botanical Laboratory,

Woolwich Polytechnic,

D. R. Chesterman.

London.

July 30 .

\footnotetext{
${ }^{1}$ Hofmeister, Jahresber. ver. Vaterl. Naturkunde, Würtemburg, 30 (1874).

2 Oltmanns, "Morphologie. u. Biologie der Algen". Band 1 (1922) •

${ }^{3}$ Langer, "Folia Crypt.", 1 (1930).

4 Maige and Nicolas, Ann. sci. nat. Bot., Sér. 9, 12 (1910).
}

\section{The Electric Quadrupole Moment of the Nucleus ${ }_{53}^{127} \mathrm{I}$}

THE hyperfine structures of many terms in the arc, as well as in the first spark, spectrum of iodine show deviations from Landé's rule ${ }^{1}$. As Murakawa has pointed out, the cause of this may be an electric quadrupole moment of the nucleus. The existence of such nuclear quadrupole moments was shown for the first time by Prof. H. Schüler and me in europium and cassiopeium ${ }^{2}$. The exact theory was given by H. Casimir ${ }^{3}$. In the case of iodine the frequent occurrence of deviations leaves no doubt that they are due to the same cause. Because the electronic structure of the terms is very complicated, most of them are not suited for a determination of the quadrupole moment. The best term is $5 p^{4} 68{ }^{4} P_{5 / 2}$ of I I measured by Tolansky. Its eigenfunction is approximately the product of the $6 s$ eigenfunction and the eigenfunction of the $5 p^{4}{ }^{3} P_{2}$ term of I II. The latter can be determined from the coarse structure, using the theory of intermediate coupling. By calculations analogous to those for bismuth ${ }^{4}$ one gets from Casimir's formula and Tolansky's measurements a value of about $-0.2 \times 10^{-24}$ for the nuclear electric quadrupole moment of iodine. That the sign is negative, one may infer also from the term $5 p^{3}\left({ }^{2} D\right) 6 s^{3} D_{2}$ measured by Murakawa.

Two remarks should be added : first, Murakawa and Tolansky have determined the spin of iodine only by means of the interval rule. As we know it fails in most terms, the value of $7 / 2$ cannot be excluded at present. Secondly, the structure of ${ }^{4} P_{5 / 2}$ of I I was deduced by Tolansky under the assumption that the combining higher levels have no perturbation. His measurements on several lines seem to show that ${ }^{4} P_{3 / 2}$ and most of the higher levels have deviations in the same direction as ${ }^{4} P_{5} / 2$. This would make the deviation in ${ }^{4} P_{5 / 2}$ somewhat greater.

All one can say at present is, that the sign of the quadrupole moment is negative and that its value is smaller than $-0.5 \times 10^{-24}$. The negative sign indicates that the charge distribution is flattened in the direction of the spin axis. It may be noted that the positive quadrupole moments now determined $\left({ }^{75} \mathrm{As},{ }^{161},{ }^{153} \mathrm{Eu},{ }^{175} \mathrm{Cp},{ }^{201} \mathrm{Hg}\right.$ ) have values ranging up to $5 \times 10^{-24}$, whereas the negative quadrupole moments $\left({ }^{63},{ }^{65} \mathrm{Cu},{ }^{127} \mathrm{I},{ }^{209} \mathrm{Bi}\right)$ all have values ${ }^{5}$ smaller than $-0.5 \times 10^{-24}$.

Note added in proof: Recently, L. A. Strait and F. A. Jenkins ${ }^{B}$ have shown by means of intensity measurements in the band spectrum that the spin of iodine is $5 / 2$.

Astrophysikalisches Observatorium,

Th. SсHмIDT.

Institut für Sonnenphysik, Potsdam.

${ }^{1}$ S. Tolansky, Proc. Roy. Soc., 152, 663 (1935); Proc. Phys. Soc., 48, 49 (1936); K. Murakawa, NATURE, 137, 1030 (1936).

${ }^{2}$ H. Schüler und Th. Schmidt, Z. Phys., 94, 457 (1935); 95, 265 (1935),

${ }^{3}$ H. Casimir, Physica, 7, 719 (1935).

H. Schüler und Th. Schmidt, Z. Phys., 99, 717 (1936).

5 H. Schüler und Th. Schmidt, Z. Phys., 98, 430 (1936).

' L. A. Strait and F. A. Jenkins, Phys. Rev., 49, 635 (1936).

IN the preceding note, which the Editor of NATURE has been good enough to let me see in advance of publication, Schmidt, following Murakawa ${ }^{1}$, suggests that the frequent occurrence of perturbations in terms of both the are and spark spectra of iodine indicate a nuclear quadrupole moment. This may be correct, but as I have shown that at least in the spark spectrum $^{2}$ term interaction perturbation occurs, and in view of the complex electronic structures involved, it is very likely that the perturbations may be due to either of the above causes or possibly both, depending upon individual terms (that is, if a quadrupole moment is assumed to exist). This fact makes caution desirable and any calculation of the quadrupole moment can only be very approximate. Schmidt has been obliged to employ my measurements for the arc spectrum, and as I have previously pointed out, the structures in this spectrum are not so well resolved as in the spark spectrum. His calculation is therefore based upon a small deviation from the interval rule in which I was not able to prove whether more than one level was affected, so that the type of perturbation is uncertain.

Regarding Schmidt's suggestion that the spin may be $7 / 2$, it may be pointed out that Murakawa ${ }^{3}$ (spark spectrum), Tolansky ${ }^{4}$ (arc spectrum, spark spectrum) and Lacroute ${ }^{5}$ (Zeeman effect) all give 5/2. This value may be considered as certain, therefore, particularly in view of the Zeeman effect support.

The approximate quadrupole moment suggested by Schmidt is of course only tentative. I am engaged now in investigating the fine structures in the ultraviolet lines of the iodine spectra with the view of obtaining further data.

Physics Department,

S. Tolansky.

University of Manchester. July 30 .

' K. Murakawa, NATURE, 137, 1030 (1936).

2 S. Tolansky, Proc. Phys. Soc., 48, 49 (1936).

' K. Murakawa, Sci. Pap. Inst. phys. chem. Res. Tokyo, 20, 285 (1933).

4 S. Tolansky, Proc. Roy. Soc., A, 149, 269 (1935); Proc. Roy. Soc.

A, 152, 663 (1935) ; Proc. Phys. Soc., 48, 49 (1936).

${ }^{5}$ P. Lacroute, Thesis (Faculty of Science, University of Paris, Nov. 1934). 